\title{
Dynamic Regulation of Spike-timing Dependent Plasticity in Electrosensory Processing
}

\author{
Patrick D. Roberts ${ }^{\mathrm{a}}$, Gerardo Lafferriere ${ }^{\mathrm{b}}$, Nathaniel Sawtell ${ }^{\mathrm{a}}$ \\ Alan Williams ${ }^{\mathrm{a}}$, Curtis C. Bell ${ }^{\mathrm{a}}$ \\ ${ }^{a}$ Neurological Sciences Institute, Oregon Health $\&$ Science University, \\ Beaverton, OR 97006 USA \\ ${ }^{\mathrm{b}}$ Department of Mathematics and Statistics, Portland State University, \\ Portland, OR 97207 USA
}

\begin{abstract}
This study investigates the control of spike-timing dependent plasticity (STDP) by regulation of the dendritic spike threshold of the postsynaptic neuron. The control of synaptic plasticity may be implemented in the electrosensory system of mormyrid electric fish by feedback control. Dendritic spikes constitute the timing signal of the STDP learning rule that regulates the output of this initial electrosensory processing structure, and the threshold of these spikes appears to be regulated by recurrent inputs from an external nucleus. However, the control dynamics must be shown to be stable, and the conditions for stability would constrain potential models of synaptic regulation. The global stability conditions for the control of STDP are derived using nonlinear control dynamical theory.
\end{abstract}

Key words: mormyrid, plasticity, modeling, electrosensory, control

\section{Introduction}

Central processes can influence how the nervous system interprets incoming sensory information. This control of sensory processing allows the nervous system to focus on important new information needed to perform specific tasks. However, it is presently unclear how sensory feedback, via central structures, affects sensory processing.

A system where centrally originating signals are well-characterized is the initial stage of sensory processing in the electrosensory system of mormyrid electric fish. This weakly electric fish is a nocturnal species that senses its environment 
by emitting a weak electric pulse and then detecting the distortions caused by external objects with electrosensory receptors in its skin. This electrosensory system requires exceptional timing and precision in order for the fish to navigate through its environment and identify external objects.

Sensory image cancellation of mormyrid electric fish. The site of initial electrosensory information processing is the electrosensory lateral line lobe (ELL), a cerebellum-like neural structure (Fig. 1A). Neurons of the ELL are affected not only by electrosensory input, but also by signals from central regions of the brain that inform the ELL about the timing of the motor command that elicits the electric pulse. In addition, the output of the ELL is fed back via an external nucleus, the preeminential nucleus $(\mathrm{PE})$, that receives input from higher processing centers. Thus, electrosensory processing is controlled by both initial processing in ELL and by higher centers.

The responses of neurons in the ELL are found to be adaptable to changing sensory conditions that effect the electrosensory system. This adaptability leads to the ability of these neurons to store a negative image of the fish's expectation of its own electrical signal. The negative image essentially predicts expected sensory input that is specific to the time course of the sensory response and its polarity (excitation or inhibition). The ELL acts as an adaptive sensory processor that cancels predictable sensory input [1], allowing unexpected input to stand out. However, the adaptive processes that lead to the prediction may be controlled by descending input from sensory feedback.

The motor command signals drive parallel fibers in ELL at various delays to about $80 \mathrm{~ms}$ [2]. Parallel fibers synapse onto apical dendrites of medium ganglion (MG) cells in the molecular layer of the ELL (Fig. 1A). MG cells are inhibitory, Purkinje-like interneurons, synapsing locally onto the efferent cells (LF in Fig. 1A), the sole output of the ELL. Electrosensory input from the periphery is transmitted to MG and efferent cells via interneurons in the deep layers of ELL.

Spike-timing dependent synaptic plasticity (STDP). Anti-Hebbian spiketiming dependent plasticity (STDP) [3] at the synapses from parallel fibers onto MG cells has been demonstrated in vitro $[1,4]$. The size and direction of changes in synaptic efficacy depend on the exact timing between pre- and postsynaptic spikes at the synapses from parallel fibers onto MG cells. MG cells have 2 types of spikes: (1) narrow, low-threshold, axon spikes that do not invade the soma, and (2) broad, high-threshold, soma-dendritic spikes that propagate into the apical dendrites [5]. The timing of dendritic spikes participates in the STDP of the parallel fiber synapse [4]. Depression of the excitatory postsynaptic potential (EPSP) was observed only after pairings in which the dendritic spike was evoked within 60 msec after the onset of the 
parallel fiber EPSP, while pairings at other delays yielded potentiation. This synaptic plasticity controls the adaptive responses of MG cells to changing sensory input so that predictable sensory images are ignored and explain the generation of negative images as observed in vivo [6,7]. Recent studies suggest that centrally generated signals actively control the threshold of dendritic spikes following the EOD motor command and, thus, control synaptic plasticity and adaptive sensory processing in the ELL. However, it is unclear how feedback loops from central regions of the brain affect the storage of sensory information and learning dynamics.

The goal of this study is to determine how learning dynamics are affected by feedback. Recent mathematical modeling work by the authors suggests that adaptation in ELL must be modulated to maintain the motor command-driven EPSP of MG cells. The model suggested that adaptation could be modulated by increasing the threshold of the dendritic spike during the motor commanddriven EPSP. Subsequent experimental work showed that there is, indeed, an increased dendritic spike threshold during the motor command-driven EPSP.

Synaptic inhibition has been found to modulate synaptic plasticity in hippocampal slices, presumably by regulating the back-propagation of dendritic spikes [8]. Inhibitory input has been observed in MG cells during the motor command. One possible source of inhibition is from cells in PE that are excited by the output of ELL. The morphology and physiology of MG cells suggests the hypothesis that inhibitory synaptic inputs to the apical dendrites, that are time-locked to the motor command, increase the threshold of dendritic spikes while excitatory inputs simultaneously excite axon spikes.

\section{Results}

This project investigates how descending inputs can control the shape of initial sensory responses by controlling synaptic plasticity, and whether that control of synaptic plasticity is stable. The problem is set up as a nonlinear control theoretic model to help elucidate how various control inputs affect the learning dynamics. The authors find that the feedback control of synaptic plasticity is stable only if the spike-timing dependent learning rule is (at least marginally) stable. In addition, it is found that stability of small perturbations around equilibrium is maintained if the feedback gain of MG cell output is small. Since the output gain is determined by noise in spike generation of the feedback circuit, then the conclusion is that noisy neural transmission in the feedback loop is required to stabilize feedback regulation of synaptic plasticity.

We will follow the development in [6] and [9] for the equations with some changes of the notation. We assume to have $N$ time steps, $x_{1}, \ldots, x_{N}$ within a single EOD cycle. For each $n=1, \ldots, N$, we let $V_{n}(t)$ denote the membrane 

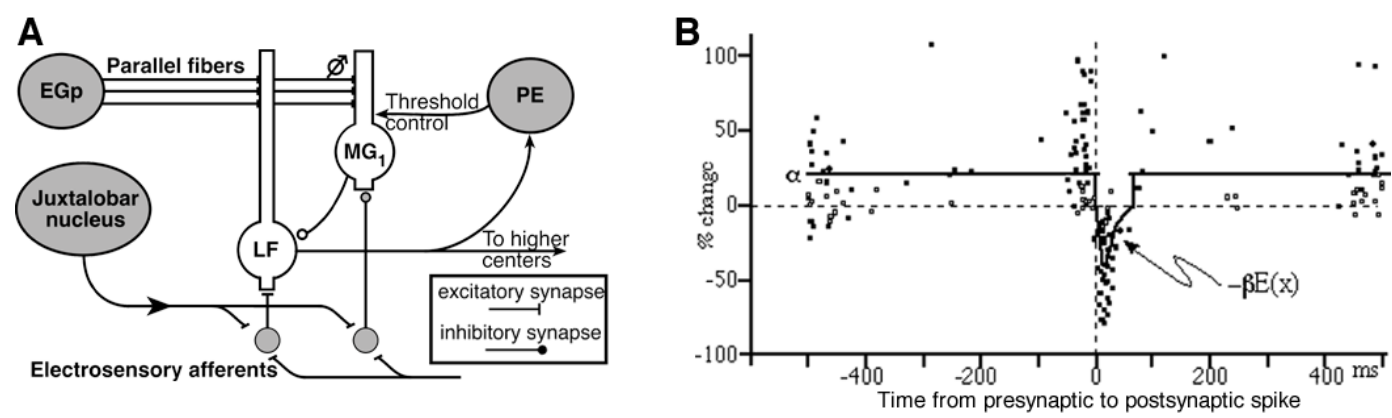

Fig. 1. Circuitry of the electrosensory lateral line lobe (ELL) with threshold control loop. (A) The electric organ corollary discharge (CD) signals enter the ELL through the eminentia granularis posterior (EGp) that gives rise to parallel fibers responding to each CD and through the juxtalobar nucleus that converges into granular cells in the deep layers of ELL with electrosensory afferents. Parallel fibers (PF) synapses excite medium ganglion $\left(\mathrm{MG}_{1}\right)$ and large fusiform (LF) cells. STDP at the $\mathrm{PF}$ synapse onto $\mathrm{MG}_{1}$ causes the output to adapt to the temporal pattern of the electrosensory image. The output of the LF cells projects to the preeminential nucleus (PE) and to higher centers of sensory processing and perception such as the telencephalon. Recurrent inputs from the PE to the deep molecular layer may control the dendritic spike threshold, and therefore synaptic plasticity.(B) Data from [4] showing STDP at the PF synapse onto $\mathrm{MG}_{1}$. The change in EPSP amplitude depends on the time between onset of the EPSP and dendritic spike peak. Depression occurs only if a dendritic spike in a $\mathrm{MG}_{1}$ follows the arrival of a parallel fiber spike within $60 \mathrm{msec}$; otherwise the synapse is potentiated.

potential of an MG cell in the ELL at time $x_{n}$ ms after the triggering of the EOD in cycle $t$. The variable $w_{n}(t)$ will denote the weights on the excitatory synapses. The interpretation is that for each $n, w_{n}(t)$ denotes the weight at the synapse from a single parallel fiber. The equations relating all these variables are as follows:

$$
V_{n}(t)=\sum_{m=1}^{N} w_{m}(t) E\left(x_{n}-x_{m}\right)+V_{n}^{e l}
$$

where $E$ is the (normalized) EPSP waveform and $V_{n}^{e l}$ is the electrosensory signal in $\mathrm{MG}_{1}$ and LF. The dynamics for the synaptic weights are $\Delta w_{n}(t)=$ $\alpha_{w}-\beta_{w} L_{w}\left(x_{b}-x_{n}\right)$, where $L_{w}$ is the learning function and $x_{b}$ denotes the time of occurrence of a broad spike following the beginning of each cycle. We treat $x_{b}$ as a random variable with a distribution encoded by the probability of a spike function, $f\left(V_{n}(t), \theta_{n}\right)=\left(1+\exp \left\{-\mu\left[V_{n}(t)-\theta_{n}\right]\right\}\right)^{-1}$, where $\mu>0$ is a noise parameter and $\theta_{n}$ is the threshold at $x_{n}$. The smaller the $\mu$ the more noise there is in the system. In the noiseless limit, as $\mu \rightarrow \infty$, the function $f$ becomes a discontinuous threshold function.

Using the probability distribution we will replace the function $\Delta w\left(x_{n}, t\right)$ in Equation (1) with its ensemble average 


$$
\left\langle\Delta w_{n}(t)\right\rangle=\alpha_{w}-\beta_{w} \sum_{p=1}^{N} L_{w}\left(x_{p}-x_{n}\right) f\left(V_{p}(t), \theta_{p}\right)
$$

The corresponding equation for $V_{n}(t)$ is

$$
V_{n}(t+1)=V_{n}(t)+\alpha_{w}-\beta_{w} \sum_{p=1}^{N}\left(R^{T} R\right)_{n p} f\left(V_{p}(t), \theta_{p}\right)
$$

where we introduced the matrix $R$ as $R_{i j}=E\left(x_{j}-x_{i}\right) \quad i, j=1, \ldots, N$ and assumed $L_{w}=E$.

\section{Control System Formulation}

We now want to look at $\Theta=\left(\theta_{1}, \ldots, \theta_{N}\right)^{T}$ as a variable whose value is to be adjusted by feedback from other nuclei. We will use the formalism from control systems theory $([10])$ to set up the equations governing a nonlinear control system with $\Theta$ as the control variable. Equation (3) in vector form becomes

$$
\vec{V}(t+1)=\vec{V}(t)+\alpha_{w} \mathbf{1}-\beta_{w} R^{T} R \vec{f}(\vec{V}(t), \Theta)
$$

where $\vec{f}(\vec{V}(t), \Theta)=\left(f\left(V_{1}(t), \theta_{1}\right), \ldots, f\left(V_{N}(t), \theta_{N}\right)\right)^{T}$. For each vector $\hat{\Theta}$ we will have an equilibrium $\hat{V}$ (in fact, $\hat{V}-\hat{\Theta}$ is a multiple of the all ones vector 1) provided $\alpha_{w} / \beta_{w}<1$ [6]. The equilibrium $\hat{V}$ is not just locally, but globally asymptotically stable as long as $\mu \beta_{w} / 8<1$. That is, global stability is guaranteed if $\mu$ is small enough (i.e. noise is sufficiently high). This can be shown via Lyapunov methods using $x^{T} P x$ as a Lyapunov function (after a translation), where $P$ is a suitable multiple of $\left(R^{T} R\right)^{-1}$. In fact, the stability results hold for much more general functions than the sigmoid $f$ defined above. All that is needed is that the function be strictly increasing and that its derivative satisfy $\beta_{w} \max f^{\prime}(x)<2$ (moreover, a different $f$ may be used on each component). This makes it possible to guarantee stability for a large class of feedback laws of the form $\theta_{i}=\hat{\theta}_{i}+g\left(V_{i}-\hat{V}_{i}\right)$ with $g(0)=0$. Indeed, consider such a law. The resulting closed loop system has the same form as (4) with $f\left(V_{i}(t), \theta_{i}\right)$ replaced by $f\left(V_{i}(t), \hat{\theta}_{i}+g\left(V_{i}-\hat{V}_{i}\right)\right)$. The derivative of the new composite function at any point has the form $\mu \bar{f}(1-\bar{f})\left(1-\overline{g^{\prime}}\right)$, where $\bar{f}$ and $\overline{g^{\prime}}$ are the values of $f$ and $g^{\prime}$ at the given point. This shows that, if $f$ is the sigmoid above satisfying the stability condition, the feedback law will not destabilize the system as long as $0<\overline{g^{\prime}}<1$ (at all points), and moreover, the system will remain globally stable.

Large values of $g$ lead to multiple equilibria, some of them unstable. Negative values of $g$ can cause oscillations around unstable equilibria while the weights remain bounded. Applicability of the model under those values of $g$ has not yet been determined empirically. In conclusion, the control loop via PE that 
allows the electrosensory system to regulate its own output, will be stable if the learning rule is stable, and also if the response properties of neurons in $\mathrm{PE}$ are such that the gain is not too great.

\section{Acknowledgments}

This material is based upon work supported by the National Institutes of Health under Grant No. R01-MH60364 (PDR) and F32-NS049728 (NS), by the National Science Foundation under Grants No. IOB-0445648 (PDR) and DMS-0408334 (GL) and by a Career Support grant from Portland State University (GL).

\section{References}

[1] C. C. Bell, A. Caputi, K. Grant, J. Serrier, Storage of a sensory pattern by anti-Hebbian synaptic plasticity in an electric fish, Proc. Natl. Acad. Sci. USA 90 (1993) 4650-4654.

[2] C. C. Bell, K. Grant, J. Serrier, Sensory processing and corollary discharge effects in the mormyromast regions of the mormyrid electrosensory lobe: I. Field potentials and cellular activity in associated structures, J. Neurophysiol. 68 (1992) 843-858.

[3] L. F. Abbott, S. B. Nelson, Synaptic plasticity: taming the beast, Nature Neurosci. (suppl.) 3 (2000) 1178-1183.

[4] C. C. Bell, V. Han, Y. Sugawara, K. Grant, Synaptic plasticity in a cerebellumlike structure depends on temporal order, Nature 387 (1997) 278-281.

[5] K. Grant, Y. Sugawara, L. Gomes, V. Han, C. C. Bell, The Mormyrid electrosensory lobe in vitro: Physiology and pharmacology of cells and circuits, J. Neurosci. 18 (1998) 6009-6025.

[6] P. D. Roberts, C. C. Bell, Computational consequences of temporally asymmetric learning rules: II. Sensory image cancellation, J. Compu. Neurosci. 9 (2000) 67-83.

[7] A. Williams, P. D. Roberts, T. K. Leen, Stability of negative-image equilibria in spike-timing-dependent plasticity, Phys Rev E 68 (2-1) (2003) 021923.

[8] P. M. Steele, M. D. Mauk, Inhibitory control of LTP and LTD: Stability of synapse strength, J. Neurophysiol. 81 (1999) 1559-1577.

[9] P. D. Roberts, Dynamics of temporal learning rules, Phys. Rev. E 62 (2000) 4077-4082.

[10] E. Sontag, Mathematical Control Theory - Deterministic Finite Dimensional Systems, 2nd Edition, Springer, New York, 1998. 